\title{
Automatic Construction of 3D Statistical Deformation Models Using Non-rigid Registration
}

\author{
D. Rueckert ${ }^{1}$, A.F. Frangi ${ }^{2,3}$, and J.A. Schnabel ${ }^{4}$ \\ 1 Visual Information Processing, Department of Computing, Imperial College, London, UK \\ dr@doc.ic.ac.uk, http://www.doc.ic.ac.uk/ /dr \\ ${ }^{2}$ Grupo de Tecnologia de las Comunicaciones, Departamento de Ingenieria Electronica y \\ Comunicaciones, Universidad de Zaragoza, Spain \\ 3 Image Sciences Institute, University Medical Center Utrecht (UMC), Utrecht, NL \\ 4 Computational Imaging Science Group, Guy's Hospital, King's College London, UK
}

\begin{abstract}
In this paper we introduce the concept of statistical deformation models (SDM) which allow the construction of average models of the anatomy and their variability. SDMs are built by performing a statistical analysis of the deformations required to map anatomical features in one subject into the corresponding features in another subject. The concept of SDMs is similar to active shape models (ASM) which capture statistical information about shapes across a population but offers several new advantages over ASMs: Firstly, SDMs can be constructed directly from images such as MR or CT without the need for segmentation which is usually a prerequisite for the construction of active shape models. Instead a non-rigid registration algorithm is used to compute the deformations required to establish correspondences between the reference subject and the subjects in the population class under investigation. Secondly, SDMs allow the construction of an atlas of the average anatomy as well as its variability across a population of subjects. Finally, SDMs take the 3D nature of the underlying anatomy into account by analysing dense $3 \mathrm{D}$ deformation fields rather than only the $2 \mathrm{D}$ surface shape of anatomical structures. We demonstrate the applicability of this new framework to MR images of the brain and show results for the construction of anatomical models from 25 different subjects.
\end{abstract}

\section{Introduction}

The significant inter-subject variability of anatomy and function makes the interpretation of medical images a very challenging task. Atlas-based approaches address this problem by defining a common reference space. Mapping data sets into this common reference space not only accounts for anatomical and functional variations of individual subjects, it also offers a powerful framework to facilitate comparison of anatomy and function over time, between subjects, between groups of subjects and across sites. Consequently, a number of different elastic [1] and fluid [2,3] warping techniques have been developed for this purpose. A recent review of different non-rigid registration techniques can be found in [4]. Traditional medical atlases contain information about anatomy and function from a single individual focusing primarily on the human brain 
[5]. Even though the individuals selected for these atlases may be considered normal, they may represent an extremum of a normal distribution. To address this problem, researchers have developed various probabilistic and statistical approaches which include information from a group of subjects making them more representative of the population under investigation [6.78].

Statistical models of shape variability [9] or Active Shape Models (ASM) have been successfully applied to perform various image analysis tasks in $2 \mathrm{D}$ and $3 \mathrm{D}$ images. In building those statistical models, a set of segmentations of the shape of interest is required as well as a set of landmarks that can be unambiguously defined in each sample shape. An extension of ASMs are the so-called Active Appearance Models (AAM) [10] which have been used for atlas matching. This model incorporates not only information about the spatial distribution of landmarks but also about the intensity distribution at the landmarks. In a different approach Wang et al. [11] suggested to use statistical shape information as priors for a non-rigid registration. A fundamental problem when building these models is the fact that it requires the determination of point correspondences between the different shapes. The manual identification of such correspondences is a time consuming and tedious task. This is particularly true in 3D where the amount of landmarks required to describe the shape accurately increases dramatically compared to $2 \mathrm{D}$ applications.

In a recent paper [12] we have described an automated way of establishing correspondences between different shapes via a non-rigid registration algorithm [13]. In this paper we present a natural extension of our previous approach in which we perform statistical analysis directly on the deformation fields required to match different anatomies. We use the term statistical deformation models (SDM) to describe this framework since it allows the construction of average models of the anatomy and their statistical variability across a population of subjects.

\section{Method}

Traditionally, landmarks are anatomically characteristic points which can be uniquely identified across a set of individuals. The goal of inter-subject registration is to find the optimal transformation $\mathbf{T}_{i}: \mathbf{x} \mapsto \mathbf{x}^{\prime}$ which maps any point $\mathbf{x}$ in the anatomy of the reference subject $\mathcal{S}_{r}$ into its corresponding point $\mathbf{x}^{\prime}$ in the anatomy of any other subject $\mathcal{S}_{i}$ in the population class. Assuming a one-to-one correspondence of anatomical structures across subjects, the registration of images between different subjects yields a dense set of so-called pseudo-landmarks. In our new framework we perform a principal component analysis (PCA) on these pseudo-landmarks which is equivalent to performing a PCA on the deformation fields required to map the anatomy of one subject into the anatomy of another subject. In the following we will describe our new framework in more detail.

\subsection{Non-rigid Registration}

In practice, the anatomical variability between subjects cannot be sufficiently explained by an affine transformation which only accounts for differences due to position, orien- 
tation and size of the anatomy. To capture the anatomical variability, it will be necessary to employ non-rigid transformations such as elastic or fluid transformations. We are using a non-rigid registration algorithm which has been previously applied successfully to a number of different registration tasks [13 14]. This algorithm uses a combined transformation $\mathbf{T}$ which consists of a global transformation and a local transformation:

$$
\mathbf{T}(\mathbf{x})=\mathbf{T}_{\text {global }}(\mathbf{x})+\mathbf{T}_{\text {local }}(\mathbf{x})
$$

The global transformation describes the overall differences between the two subjects and is represented by an affine transformation. The local transformation describes any local deformation required to match the anatomies of the subjects. We have chosen a free-form deformation (FFD) model based on B-splines which is a powerful tool for modelling 3D deformable objects. The basic idea of FFDs is to deform an object by manipulating an underlying mesh of control points. The resulting deformation controls the shape of the $3 \mathrm{D}$ object and can be written as the $3 \mathrm{D}$ tensor product of the familiar 1D cubic B-splines,

$$
\mathbf{T}_{\text {local }}(\mathbf{x})=\sum_{l=0}^{3} \sum_{m=0}^{3} \sum_{n=0}^{3} B_{l}(u) B_{m}(v) B_{n}(w) \mathbf{c}_{i+l, j+m, k+n}
$$

where $\mathbf{c}$ denotes the control points which parameterise the transformation. The optimal transformation is found by minimising a cost function associated with the global transformation parameters as well as the local transformation parameters. The cost function comprises two competing goals: The first term represents the cost associated with the voxel-based similarity measure, in this case normalised mutual information [15], while the second term corresponds to a regularization term which constrains the transformation to be smooth [13].

The resulting transformation $\mathbf{T}$ maps each point in the anatomy of the reference subject $S_{r}$ to the corresponding point in the anatomy of subject $S$. Since the transformation $\mathbf{T}$ is a sum (rather than a concatenation) of a global and local transformation, we need to remove any dependency of the local transformation on the global transformation (which is a result of the different position, orientation and size of each subject's anatomy):

$$
\mathbf{x}+\mathbf{d}(\mathbf{x})=\mathbf{T}_{\text {global }}^{-1}\left(\mathbf{T}_{\text {global }}(\mathbf{x})+\mathbf{T}_{\text {local }}(\mathbf{x})\right)
$$

Thus, the deformation $\mathbf{d}$ describes the anatomical variability across the population class in the coordinate system of the reference subject.

\subsection{Construction of Statistical Deformation Models}

The concept of statistical deformation models (SDMs) is closely related to the idea of ASMs. The key difference is that we apply a principal component analysis (PCA) to the deformation fields required to map one anatomy to another anatomy rather than to corresponding points: Suppose that we have $n$ deformation fields described as vectors $\mathbf{d}_{i}$. These deformation fields are the result of the non-rigid registration algorithm described in the previous section and map the anatomy of the reference subject $\mathcal{S}_{r}$ into the 
anatomy of the other individuals $\mathcal{S}_{i}$ in the population class under investigation. Each deformation field $\mathbf{d}_{i}$ can be expressed as a concatenation of $m$ 3-D vectors which describe the deformation at each voxel in the image of the reference subject. The goal of SDMs is to approximate the distribution of $\mathbf{d}$ using a parameterised linear model of the form

$$
\mathbf{d}=\hat{\mathbf{d}}+\Phi \mathbf{b}
$$

where $\hat{\mathbf{d}}$ is the average deformation field for all $n$ subjects,

$$
\hat{\mathbf{d}}=\frac{1}{n} \sum_{i=1}^{n} \mathbf{d}_{i}
$$

and $\mathbf{b}$ is the model parameter vector. The columns of the matrix $\boldsymbol{\Phi}$ are formed by the principal components of the covariance matrix $\mathbf{S}$ :

$$
\mathbf{S}=\frac{1}{n-1} \sum_{i=1}^{n}\left(\mathbf{d}_{i}-\hat{\mathbf{d}}\right)\left(\mathbf{d}_{i}-\hat{\mathbf{d}}\right)^{T}
$$

From this, we can calculate the principal modes of variation of the deformation field as the eigenvectors $\phi_{i}$ and corresponding eigenvalues $\lambda_{i}$ (sorted so that $\lambda_{i} \geq \lambda_{i+1}$ ) of $\mathbf{S}$.

\subsection{Interpretation of Statistical Deformation Models}

A common problem encountered during the construction of a model of the average anatomy is the choice of the reference subject to which all other subjects are registered. Even though the reference subject selected may be considered normal, it may represent an extremum of a normal distribution. Assuming a perfect registration, the registration will align the anatomy of each subject with the anatomy of the reference subject. Thus, the average model will be constructed in the coordinate system of the reference subject. To remove any bias of the average model towards a particular anatomy, we can construct the average model of the anatomy in its natural coordinate system. This natural coordinate system is the coordinate system which requires the least residual deformation to explain the anatomical variability across all individuals. Based on a point $\mathbf{x}$ in the space of the reference subject we can find the corresponding point $\mathrm{x}^{\prime}$ in its natural coordinates by applying the average deformation vector $\hat{\mathbf{d}}$ :

$$
\mathbf{x}^{\prime}=\mathbf{x}+\hat{\mathbf{d}}(\mathbf{x})
$$

Within this natural coordinate system we can now study the anatomical variability of the population class under investigation. Recall that in eq. (4) the vector $\mathbf{b}$ provides a parameterisation of the deformations in terms of its principal modes. Varying the parameter vector $\mathbf{b}$ will generate different instances of the deformation field. By applying these deformation fields to the average anatomy we can generate instances of the class of anatomy under analysis. Under the assumption that the cloud of deformation vectors at each point follows a multi-dimensional Gaussian distribution, the variance of the $\mathrm{i}$-th parameter, $b_{i}$, across the population is given by eigenvalue $\lambda_{i}$. By applying limits to the variation of $b_{i}$, for instance $\left|b_{i}\right| \leq \pm 3 \sqrt{\lambda_{i}}$, it can be ensured that a generated anatomy is similar to the anatomies in the training class. 


\begin{tabular}{|l|c|c|}
\hline Registration & L. nucleus caudate & R. nucleus caudate \\
\hline Affine & $48.86 \%$ & $41.35 \%$ \\
\hline Non-rigid $(20 \mathrm{~mm})$ & $62.56 \%$ & $58.98 \%$ \\
\hline Non-rigid $(10 \mathrm{~mm})$ & $75.01 \%$ & $74.75 \%$ \\
\hline Non-rigid $(5 \mathrm{~mm})$ & $86.73 \%$ & $85.65 \%$ \\
\hline
\end{tabular}

Table 1. Average overlap between the left and right nucleus caudate of the reference subject after registration with all other individuals.

\section{Results}

To demonstrate our approach we have used 25 brain MR images from different subjects with schizophrenia to construct a statistical deformation model of the brain. All images were acquired at the Department of Psychiatry of the University Medical Center Utrecht using a $3 \mathrm{D}$ FFE sequence $\left(\mathrm{TE}=4.6 \mathrm{~ms}, \mathrm{TR}=30 \mathrm{~ms}\right.$, flip angle $\left.=30^{\circ}\right)$ on a $1.5 \mathrm{~T} \mathrm{MR}$ imaging system (Philips Gyroscan ACS-NT). These images have a voxel size of $1 \times 1 \times$ $1.2 \mathrm{~mm}^{3}$ and $200 \times 200 \times 160$ voxels. Out of the 25 subjects we have randomly selected one individual which was used as the reference subject. We have then registered each of the remaining 24 individuals to this reference subject. For the non-rigid registration we have used control point spacings of $20 \mathrm{~mm}, 10 \mathrm{~mm}$ and finally $5 \mathrm{~mm}$ [13]14]. In order to assess the quality of the inter-subject registration, we have calculated the overlap between a manually segmented deep structure in the brain, the nucleus caudate, after affine and non-rigid registration. The results summarised in Table 1 show that after non-rigid registration the average overlap between the nucleus caudate of the reference subject and those of the remaining 24 individuals is more than $85 \%$. Given the small size of the nucleus caudate this indicates a very good registration.

The first step of the construction of statistical deformation models is to build a model of the average anatomy of all subjects after non-rigid registration. The resulting atlas constructed in the coordinate system of the reference subject is shown in the top row of Figure 1 in form of an average intensity image 1 . In the second step we have removed any bias of the atlas towards the reference subject by applying the average deformation $\hat{\mathbf{d}}$ to the atlas. The resulting atlas in its natural coordinate system is shown in the bottom row of Figure 1 In this example we can clearly see that the average reproduces the large ventricular system of the subject chosen as reference subject. Removing any bias of the atlas towards the reference subject yields an average model in which the ventricular system is significantly smaller.

The final step of the construction of statistical deformation models is the calculation of the principal modes of variation of the deformation field which give an indication of the anatomical variability across the population under investigation. We can generate the modes of variation by varying the shape parameter $b_{i}$ and applying the resulting deformation field to the atlas. An example of the first three modes of variation is shown in Figure 2 Note, that in contrast to AAMs [10], our model does not incorporate any statistical information about intensities so that the intensities of the atlas are not affected

\footnotetext{
${ }^{1}$ Animated versions of Figure 1 and 2 are available at http://www.doc.ic.ac.uk/ dr/MICCAI01.
} 

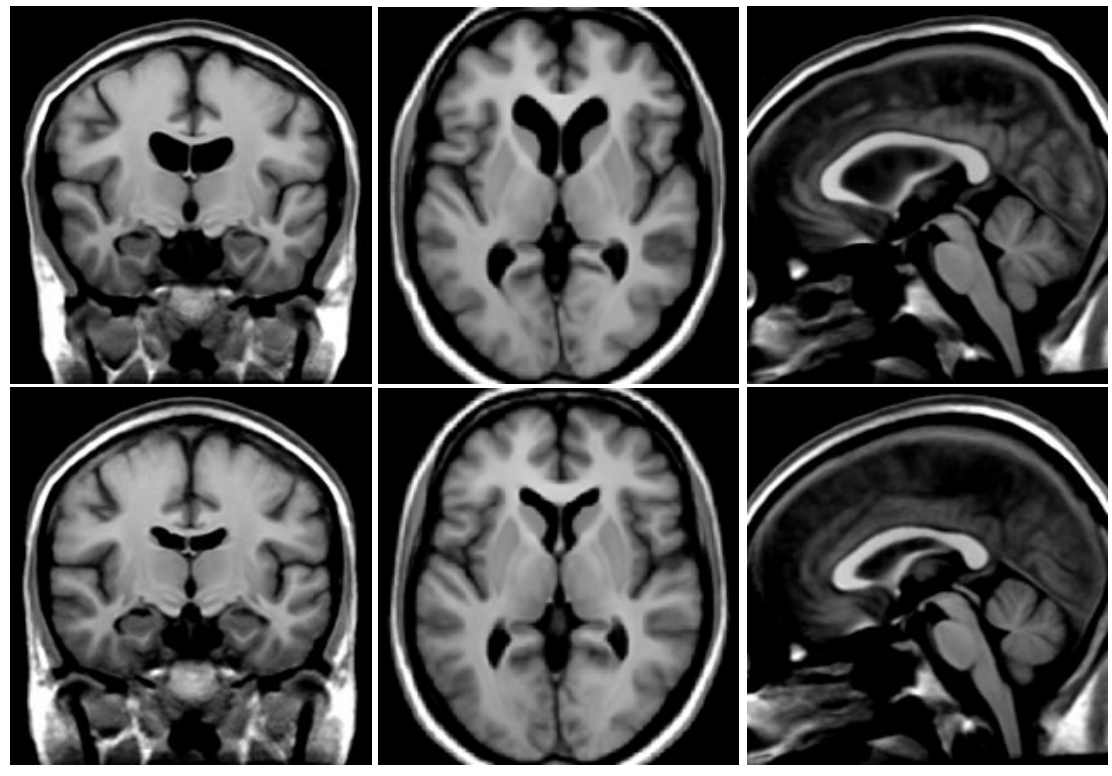

Fig. 1. The top row shows a coronal, axial and sagittal view of the average brain atlas of 25 different subjects constructed in the space of the reference subject. The bottom row shows the coronal, axial and sagittal view of the average brain atlas of 25 different subjects constructed in natural atlas coordinates. This coordinate system is obtained by applying the average deformation $\hat{\mathbf{d}}$ to the coordinate system of the reference subject.

by the modes of variation. The total number of modes of variation corresponds to the number of individuals in the population class excluding the reference subject. In our example we have 24 different modes of variation of which the first 10 modes explain more than $70 \%$ of the cumulative anatomical variability while the first 18 modes explain more than $90 \%$ of this variability.

The interpretation of the modes of variation is difficult since they are not only the result of the variability of a single anatomical structure but the result of the variability of a large number of different anatomical structures as well as their inter-relationship. The interpretation is further complicated by the fact that a dense deformation field requires performing a PCA on a very high dimensional space $(200 \times 200 \times 160=6.4$ million deformation vectors). However, we can significantly reduce the dimensionality by performing the PCA directly on the control points of the FFD $(40 \times 40 \times 32=51200$ control points). This provides a very compact representation of deformation fields using B-spline basis functions. In future experiments we are planning to use a larger number of subjects to allow a robust statistical analysis of the deformation fields.

\section{Discussion and Conclusions}

In this paper we have presented a new method for the automatic construction of statistical deformation models (SDM). These models can be used to build an atlas of the 


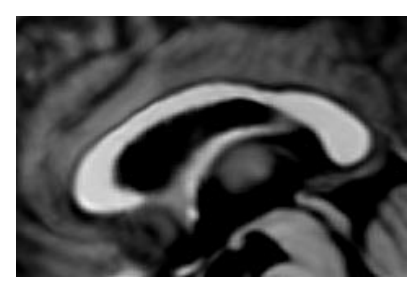

1st mode

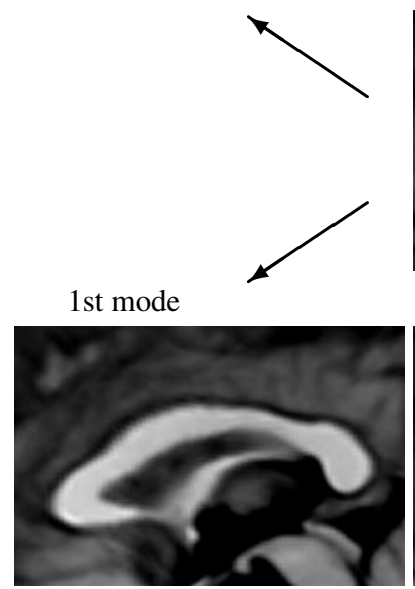

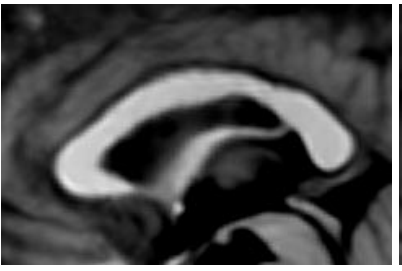

$4 \quad$ 2nd mode
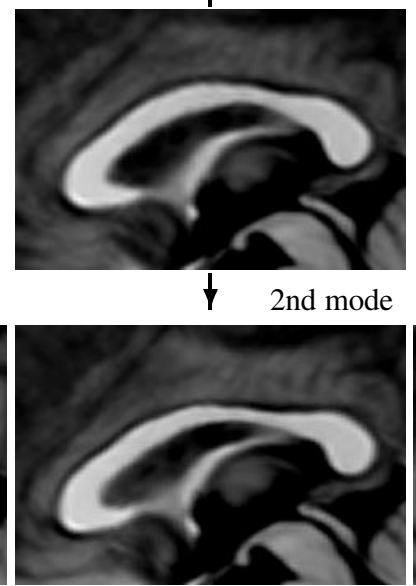

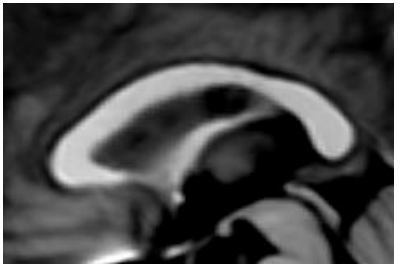

3rd mode

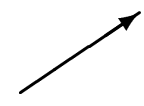

3rd mode

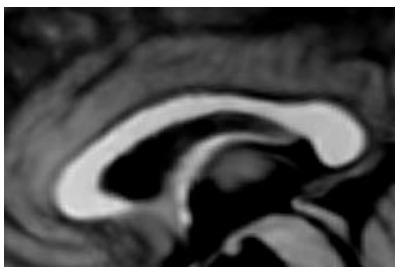

Fig. 2. Instances of the statistical deformation model showing the corpus callosum: Each image has been generated by varying the first three modes of variation between $-3 \sqrt{\lambda_{i}}$ (top row) and $+3 \sqrt{\lambda_{i}}$ (bottom row). The middle row corresponds to the average model.

average anatomy as well as its variability. This is achieved by performing a principal component analysis of the deformations required to map the anatomy of a reference subject to all other subjects in the population. A similar statistical analysis of deformation fields has been proposed by Gee et al. [7]. The key difference to the work by Gee et al. is the fact that our approach exploits the compact parameterization of the deformation fields by the B-spline representation. Our method can also be used for the construction of "stable" anatomical models which are not dependent on the choice of the reference subject. A related approach has been pursued by Guimond et al. [8]. Their approach also uses average deformations to construct an atlas of the shape and intensity. The advantage of the framework presented in this paper stems from the fact that the proposed framework allows not only to construct an atlas of the average anatomy but also a model of the principal modes of variation of this anatomy. This may be used to compare the morphometrics of an individual with those of a group or across different groups of individuals.

In practice inter-subject registration is a very challenging task and will lead to registration errors. In previous experiments we have found that our algorithm yields a registration accuracy between 1 and $2 \mathrm{~mm}$ when comparing different anatomical landmarks across individuals. This compares to an intra-observer variability between 0.2 and 0.9 
$\mathrm{mm}$ for the localisation of anatomical landmarks. We are currently investigating the effect of registration errors on the statistical analysis of the deformation fields in more detail. We are also aiming to incorporate knowledge about landmarks into the registration algorithm.

\section{Acknowledgements}

DR was partially supported by EPSRC GR/N/24919. AF was partially supported by CICYT-FEDER (2FD97-1197-C02-01). JAS is supported by Philips Medical Systems EV-AD. We would like to thank the Department of Psychiatry of the University Medical Center Utrecht for providing the data.

\section{References}

1. J. Gee, M. Reivich, and R. Bajcsy. Elastically deforming 3D atlas to match anatomical brain images. Journal of Computer Assisted Tomography, 17(2):225-236, 1993.

2. G. E. Christensen, R. D. Rabbitt, and M. I. Miller. Deformable templates using large deformation kinematics. IEEE Transactions on Image Processing, 5(10):1435-1447, 1996.

3. M. Bro-Nielsen and C. Gramkow. Fast fluid registration of medical images. In Proc. Visualization in Biomedical Computing (VBC'96), pages 267-276, 1996.

4. D. Rueckert. Non-rigid registration: Techniques and applications. In D. J. Hawkes, D. L. G. Hill, and J. Hajnal, editors, Medical Image Registration. CRC Press, 2001.

5. J. Talairach and P. Tournoux. Co-Planar Stereotactic Atlas of the Human Brain: 3Dimensional Proportional System: An Approach to Cerebral Imaging. Stuttgart, 1988.

6. P. Thompson and A. W. Toga. Detection, visualization and animation of abnormal anatomic structure with a deformable probabilistic atlas based on random vector field transformations. Medical Image Analysis, 1(4):271-294, 1997.

7. J. C. Gee and R. K. Bajcsy. Elastic Matching: Continuum Mechnanical and Probabilistic Analysis. In A. W. Toga, editor, Brain Warping, pages 183-197. Academic Press, 1999.

8. A. Guimond, J. Meunier, and J.-P. Thirion. Average brain models. Computer Vision and Image Understanding, 77:192-210, 2000.

9. T. F. Cootes, C. J. Taylor, D. H. Cooper, and J. Graham. Active Shape Models - their training and application. Computer Vision and Image Understanding, 61(1):38-59, 1995.

10. T. F. Cootes, C. Beeston, G. J. Edwards, and C. J. Taylor. A unified framework for atlas matching using active appearance models. In Proc. Information Processing in Medical Imaging (IPMI'99), pages 322-333, 1999.

11. Y. Wang and L. H. Staib. Elastic model based non-rigid registration incorporating statistical shape information. In Proc. MICCAI '98, pages 1162-1173, 1998.

12. A. F. Frangi, D. Rueckert, J. A. Schnabel, and W. J. Niessen. Automatic 3D ASM construction via atlas-based landmarking and volumetric elastic registration. In Proc. Information Processing in Medical Imaging: (IPMI'01), pages 78-91, 2001.

13. D. Rueckert, L. I. Sonoda, C. Hayes, D. L. G. Hill, M. O. Leach, and D. J. Hawkes. Nonrigid registration using free-form deformations: Application to breast MR images. IEEE Transactions on Medical Imaging, 18(8):712-721, 1999.

14. J. A. Schnabel, D. Rueckert, M. Quist, J. M. Blackall, A. D. Castellano Smith, T. Hartkens, G. P. Penney, W. A. Hall, H. Liu, C. L. Truwit, F. A. Gerritsen, D. L. G. Hill and D. J. Hawkes. A Generic Framework for Non-Rigid Registration Based on Non-Uniform MultiLevel Free-Form Deformations. In Proc. MICCAI '01, 2001. In press.

15. C. Studholme, D. L. G. Hill, and D. J. Hawkes. An overlap invariant entropy measure of 3D medical image alignment. Pattern Recognition, 32(1):71-86, 1998. 